\title{
Carácter formativo y pedagógico de la revisión de literatura en la investigación
}

\author{
Guillermo Meza-Salcedo, Gustavo A. Rubio-Rodríguez ${ }^{2}$ *, Leidy X. Mesa ${ }^{3}$ y Alexander Blandón ${ }^{4}$ \\ (1) Programa de Licenciatura en Educación Infantil, Corporación Universitaria Minuto de Dios, Calle 87 No. 20-98, \\ Ibagué, Colombia (correo-e: guillermo.meza@uniminuto.edu) \\ (2) Programa de Contaduría pública, Corporación Universitaria Minuto de Dios, Calle 87 No. 20-98, Ibagué, Colombia \\ (correo-e: gustavo.rubio-r@uniminuto.edu.co) \\ (3) Programa de Licenciatura en Educación Infantil, Corporación Universitaria Minuto de Dios, Calle 87 No. 20-98, \\ lbagué, Colombia (correo-e: 1mesa1@uniminuto.edu.co) \\ (4) Facultad Ciencias Económicas y Administrativas, Universidad del Tolima, Calle 42 \#1B-1 Barrio Santa Helena Parte \\ Alta Ibagué, Colombia (correo-e: ablandonl@ut.edu.co)
}

* Autor a quien debe ser dirigida la correspondencia

Recibido Mar. 31, 2020; Aceptado Jun. 1, 2020; Versión final Jul. 25, 2020, Publicado Oct. 2020

\begin{abstract}
Resumen
El presente artículo de reflexión enseña la importancia que representa la revisión de la literatura en la investigación como proceso formativo, que visibiliza lo que se trabaja o ha sido trabajado en determinada área de conocimiento, al interior de un ejercicio investigativo. Metodológicamente, este manuscrito se construyó desde una revisión documental, matizando aspectos intrínsecos señalados por diferentes autores sobre el tema objeto de estudio. La reflexión radica en presentar axiológicamente la preponderancia que subroga la revisión de literatura en cada una de las fases del proceso investigativo: 1) planeación o preparatoria, 2) descriptiva, 3) interpretación y análisis, 4) construcción teórica, y 5) socialización. Como conclusión, se formula que una metodología de contextualización, clasificación y categorización tiene su origen en la revisión de literatura. Esto implica investigar a partir de ella, no como suceso aislado, sino, bajo una secuencialidad y ciclicidad en las etapas que la componen.
\end{abstract}

Palabras clave: revisión de literatura; investigación formativa; metodología; pedagogía

\section{Formative and pedagogical character of the literature review in research}

\begin{abstract}
This article shows the importance of literature reviews as a training process for conducting research. Literature reviews make visible previous work in a certain area of knowledge. A documentary review was constructed from intrinsic aspects highlighted by different authors in their respective study subjects. This resulted in an axiological presentation, a reflection, on the preponderance of the literature review in each of the phases of the research process: 1) planning, 2) description, 3) interpretation and analysis, 4) theoretical construction, and 5) socialization. It is concluded that a contextualization, classification, and categorization methodology originate in the literature review. This implies conducting research starting from it, not as an isolated event, but should be part of the cyclical step-by-step phases that compose the research process.
\end{abstract}

Keywords: literature review; formative research; methodology; pedagogy 


\section{INTRODUCCIÓN}

En el ejercicio investigativo, cuando se quiere formular un anteproyecto o proyecto de investigación, es relevante una revisión metódica y pormenorizada de estudios, informes, ensayo, tesis, libros, artículos, entre otros documentos (Berns et al., 2018; Palmatier et al., 2018; Akerlind, 2005). Es decir, se requiere una exploración documental de la producción científica existente respecto al tema o cuestión que se pretende investigar (Orozco y Díaz 2018; Guevara, 2016; Huergo, 2015; Huber et al., 2014). Dicha revisión intenta contextualizar el interés investigativo, así como poner al día al investigador sobre lo que circula en el medio académico con relación a su objeto de estudio. En este sentido, dentro del trabajo investigativo, el desarrollo de la 'Revisión de Literatura' (RL) es una de las acciones fundamentales que cada investigador debe tener en cuenta en su labor, como un ejercicio de "ir tras las huellas" de su objeto de estudio.

El simple gusto del investigador frente a una temática, la conveniencia personal o grupal de una organización o empresa, la pertinencia social, así como la trascendencia teórica, la innovación y/o transformación, son algunos de los motivos que lo impulsan a lanzarse en el trabajo inagotable de la investigación. Otros motivos son también la carencia o necesidad de algo, una oportunidad, un problema que requiere ser resuelto, el cual se identifica en el diagnóstico de la investigación, incluso en la misma RL. Por eso, de acuerdo con Olson et al. (2017), intereses, temas, preguntas y problemas son los cuatro elementos que otorgan al investigador el punto de partida para dedicar su tiempo a la investigación y le posibilitan convertirse en un hábil investigador.

Sin embargo, el ejercicio investigativo en el ámbito universitario no es una tarea fácil para los estudiantes ni para los docentes: ensayar nuevas miradas en sus disciplinas, atreverse a plantear problemas, fomentar la creatividad, la reflexión y la argumentación, así como hacer ciencia en condiciones de bajo desarrollo económico y social, sigue siendo un desafío (Rojas 2008; Ortiz et al., 2012; Díaz et al., 2017). Al iniciarse en la investigación mediante la búsqueda y análisis de estudios arbitrados, los estudiantes experimentan miedo e inseguridad por su inexperiencia y por el arduo trabajo de lectura que requiere, el cual puede interferir con sus otras actividades académicas (Tan, 2007). Además, hay en ellos una baja actitud hacia la investigación como lo demuestra el estudio realizado por Rojas y Méndez (2017), así como dificultades para escribir propuestas de investigación para sus disertaciones o tesis de grado (Talinbe y Owusu-Ansah, 2014) y bajos niveles en hacer búsquedas bibliográficas y el uso de bases de datos especializadas, pues los estudiantes utilizan cuando mucho Google Académico (Rubio et al., 2018). Aunado a lo anterior, la formación para la investigación que se establece en las mallas curriculares con cursos de fundamentos o metodología de la investigación está teniendo una baja incidencia en la formación profesional (Rojas y Méndez, 2017).

Frente a estas situaciones universitarias complejas, Rojas (2008) señala que la investigación debería ser formativa y un aporte en las prácticas pedagógicas del docente, la cual ha de contribuir a la investigación científica formando jóvenes investigadores que se vincularán a las comunidades científicas, así como para el desarrollo de la ciencia y la tecnología orientada al desarrollo y la transformación social. En este sentido, la investigación formativa "[...] aparece como problema pedagógico y didáctico orientado a la aplicación de estrategias de enseñanza y de aprendizaje por descubrimiento y construcción" (p. 140 ), en la que una RL puede ser el punto de partida en tanto propicia la familiaridad del estudiante con autores, métodos y metodologías de investigación.

En efecto, la investigación encarna una lógica formativa y pedagógica en cuanto que, en el desarrollo de ésta, se estimulan las disposiciones humanas para conseguir un fin determinado, es decir, se potencializan habilidades, capacidades, destrezas, hábitos, valores, entre otros aspectos (Snyder, 2019; Orozco y Díaz, 2018; Viteri y Vázquez, 2016), los cuales se ponen en interacción tanto con el entorno como con otras personas con el propósito de que lo aprehendido se aplique en el mejoramiento de la sociedad desde diferentes capacidades dinámicas de aprendizaje (Gallego y Araque, 2019). Por tanto, en la investigación como ejercicio formativo en el que se actualiza y recrea la tradición científica (Sánchez, 2014), existe una lógica formativa que subyace a la RL mediada por la óptica del investigador (Jones et al., 2017).

Se entiende el aspecto formativo y pedagógico de la investigación, en cuanto la pedagogía está orientada a reconocer las condiciones para ampliar el espacio y enriquecer la actividad consciente del individuo, elevando su capacidad de producción intelectual y de interpretación simbólica (Sánchez, 2017; Viteri y Vázquez, 2016; Bettencourt y Houston, 2001). "El conocimiento no es la simple copia de las cosas, sino, su construcción interior; por ello, la pedagogía tiene que fundarse en la actividad del aprendiz" (Flórez, 1997, p. 4). Así, al adquirir o construir un nuevo conocimiento en el que generalmente entra en juego la $R L$, ésta se puede comprender como una tarea pedagógica en sí misma, la cual está vinculada a la formación para la investigación y propicia el inicio para una "formación de investigadores", que prepara a los profesionales para la generación y aplicación de conocimientos fuera del ámbito universitario (Pradhan y Pradhan, 2015; Rojas, 2008). Además, desde este prisma formativo de la investigación en relación con la $R L$, Randolph (2008) 
expresa que una revisión documental es una de las primeras actividades de carácter investigativo y formativo con la cual el investigador devela el pasado y presente en relación con un fenómeno estudiado.

¿Qué papel juega, entonces, la RL en la investigación formativa, así como en el desarrollo del mismo proceso de la investigación? ¿Qué habilidades investigativas se pueden potencializar con la $R L$ en los estudios de pregrado? Estos cuestionamientos orientan este artículo reconociendo que, el investigador reconfigura y recrea lo encontrado y seleccionado en la $R L$ desde su apuesta paradigmática, es decir, desde su intencionalidad. El investigador entabla un diálogo con los planteamientos de otros investigadores que se encuentra en los diversos documentos para comprender y producir 'recomprensiones', sobre la forma como una temática ha sido abordada en un tiempo y espacio determinado. Analiza quién ha dicho tal o cual cosa, cómo lo ha expresado, por qué lo ha dicho, en dónde lo ha dicho, en qué contexto lo ha dicho, dónde se encuentra esa información, cuáles son las fuentes claves y las teorías subyacentes, entre otras preguntas a las que responde una $\mathrm{RL}$ (Guirao, 2015).

\section{METODOLOGÍA}

Este trabajo se sustenta en una indagación de corte bibliográfico y documental, de tipo exploratorio. La base para el desarrollo de éste corresponde a un ensayo argumentativo, donde la búsqueda de diferentes corrientes literarias permite reflexionar y analizar, desde una perspectiva personal, lo explorado e imaginable, para entender y responder a los elementos que componen un proceso investigativo. La metodología utilizada en otros trabajos del mismo enfoque, se apoya en Snyder (2019) quien defiende la importancia de recolectar información sistemáticamente de diferentes fuentes, para fortalecer con elementos de juicio la posición del investigador. Asimismo, Mendoza y Jaramillo (2006) expresan que un ensayo argumentativo destaca los aspectos subjetivos que expresa el ensayista sobre un determinado tema, y que una de sus funciones es evidenciar el carácter interpretativo de la subjetividad, para conectar a los lectores con un horizonte de reflexión.

La relevancia de la metodología empleada se fundamenta en que la sistematización de la literatura forja la naturaleza y la esencia de los ejercicios de carácter reflexivo y argumentativo; ahondar en temáticas que generan conocimientos, coadyuvan en el entendimiento y el desarrollo de diferentes conceptos. A partir de esta especificidad, la metodología permite realizar el abordaje y la organización de la generación y actualización de productos académicos (Posada, 2017); además, concede la clasificación de la información a partir de los intereses que beneficien al investigador, asumiendo diversas intenciones "pasando por el rastreo, registro, sistematización e interpretación" (Gómez et al., 2015). Estos propósitos se enmarcan en los que justamente se pretenden abordar desde este manuscrito.

Con base en lo anterior, se hizo un rastreo exploratorio en algunas bases de datos como Scielo, WOS y Scopus, así como en el buscador Google Scholar, utilizando las categorías: revisión de literatura, revisión sistemática, revisión documental, investigación formativa, investigación en la educación superior. El criterio de elección de estas bases de datos, obedece al alto factor de impacto que han alcanzado en los diferentes sistemas de indexación, especialmente Scopus y WOS, que albergan un elevado número de revistas que contienen artículos de relevancia a nivel mundial. Una vez elegidos los documentos, se elaboraron los diferentes análisis de manera descriptiva a partir de las categorías enunciadas. De igual forma, desde la perspectiva pedagógica se eligió la información consentida, que entraña desde la $R L$ el desarrollo de un proyecto como la misma metodología de investigación.

Finalmente, las reflexiones contendidas en este manuscrito tienen su origen en dos ejercicios académicoinvestigativos en el ámbito universitario: el desarrollo de un proyecto de revisión documental sobre la ética de la investigación en las ciencias sociales, así como el acompañamiento formativo a dos semilleros de investigación, proceso que invitó a la reflexión sobre el papel de la $R L$ en la formación pedagógica universitaria.

\section{RESULTADO Y DISCUSIÓN}

Desde el acercamiento a diversas investigaciones que han desarrollado revisión de literatura y han reflexionado acerca de ésta, se enuncian las siguientes ideas que coligen su concepto: Según Kitchenham (2004), la RL es una manera de clasificar, interpretar y evaluar la investigación disponible respecto a un área o fenómeno de interés determinado. Para Gómez et al. (2015) se trata de una metodología de investigación. Y para Rodríguez y Acosta (2007) y Posada (2017), la RL es una modalidad de investigación documental en la que se produce un "perfil crítico documental actual" sobre un tema, y permite, además, "[...] analizar las dimensiones relacionales y posiciones estratégicas que caracterizan y constituyen un campo del saber" (Rodríguez y Acosta, 2007). 
Por otro lado, Gómez y Sepúlveda (2008), la conciben como una herramienta o estrategia de investigación que puede visibilizar lo que se conoce o ha sido trabajado sobre una temática o campo de conocimiento. Se trata de una investigación en sí misma que abre camino a nuevas investigaciones (Randolph, 2009). Caminar sobre lo caminado en procura de dejar su propia huella. La RL consiste en un diagnóstico ¿dónde estamos? y un pronóstico ¿dónde podemos estar? (Hoyos, 2000; Guevara, 2016). De igual forma, se define como una investigación desde la óptica de "ir tras las huellas" de un campo de conocimiento para ahondar en él, para mirar cómo ha sido abordado y cuáles son sus tendencias (Barbosa et al., 2013).

Así pues, se puede referir a la RL como "estado del conocimiento o de la cuestión", como "una investigación documental" o "una investigación sobre lo investigado", incluso como los "antecedentes de una investigación", como "minería de datos", entre otras ideas. La RL permite evidenciar enfoques y tendencias teóricas, epistemológicas y metodológicas en los diversos campos temáticos, aún más en el campo de la investigación social, la cual demanda de nuevas metodologías y abordajes para transitar por esos caminos inéditos. Camino que se emprende generalmente desde unos saberes a los que el investigador previamente ha tenido acceso, es decir, de unos saberes acumulados (Jiménez, 2006).

Para Palmatier et al. (2018), Posada (2017), Guevara (2016) y Huber et al. (2014), la RL hace referencia a "una investigación documental", que sirve de punto de partida para trascender desde la reflexión del conocimiento atesorado sobre un campo de estudio o sobre un objeto determinado. No se trata solo de desenterrar información para transmitirla, sino, de caminar sobre el rastro que otros investigadores han dejado, explorando sus huellas para beneficiarse así de toda su obra. En este sentido y de manera poética se puede expresar que, en la aventura por los bosques, cascadas y caminos de la investigación, el "investigador es un explorador que camina siguiendo el rastro, las huellas, los vestigios de otros como él, para dejar sus propias huellas" (Pérez, 2004, p. 22). Camino de investigación que otros ya han recorrido y que Booth et al. (2001) llaman "sendero bibliográfico", el cual está lleno de huellas de conocimiento a partir de las cuales "se podrá encontrar un camino a través del territorio de investigación más difícil porque una fuente [huella] siempre lleva a la otra" (p. 91).

En consecuencia, la RL es una investigación sobre lo conocido de la producción documental -teórica o metodológica- que existe sobre una temática, la cual trasciende la recopilación y la sistematización de los materiales encontrados (Hult, 2015). Esta trascendencia se da a partir de descubrir dinámicas y lógicas presentes en las fuentes documentales y después de su comprensión hermenéutica, pues la $R L$ conduce a formular hipótesis y propuestas de acción referidas a esa área particular del conocimiento que pueden ser utilizados por la comunidad académica (Orozco y Díaz, 2018; Sorescu et al., 2017).

Además, la RL se puede entenderse como un trabajo de "artesanía intelectual" que implica unos saberes prácticos y operativos. Requiere del desarrollo de varios ejercicios procedimentales: de búsqueda, de clasificación, de categorización, de conceptualización, de análisis, entre otros. Éstos se proponen reconocer y rescatar la pluralidad epistemológica, teórica, metodológica y técnica de las investigaciones, determinando conexiones, complementariedades, diferencias, límites y umbrales. Además, para evidenciar la multiplicidad y variedad de la producción presente en los materiales analizados (Barattucci y Bocciolesi, 2018; Berns et al., 2018). Hoyos (2000), concibe la RL como un "proceso en forma espiral" sobre un fenómeno escogido, a partir de un interés particular, ya sea por sus implicaciones sociales o culturales. Este fenómeno es investigado a través de la producción teórica existente, la cual lo enfoca, describe y contextualiza desde la multidisciplinariedad o interdisciplinariedad, y desde lo teórico, metodológico e instrumental. Además, manifiesta que la finalidad fundamental de la $R L$ es "dar cuenta de construcciones de sentido sobre datos que apoyan un diagnóstico y un pronóstico en relación con el material documental sometido a análisis" (p. 57).

\section{Propósitos de la revisión de literatura}

Al acercarse a la $\mathrm{RL}$ como un estudio para recuperar sistemáticamente un cierto tipo de información o de datos, se pueden destacar algunos objetivos como el de indagar y recopilar información acerca de lo que se ha elaborado en torno a un objeto de estudio, para así establecer un balance de las investigaciones de forma temática y cronológica (Berns et al., 2018; Gómez y Sepúlveda, 2008), pero también para vislumbrar hacia dónde pueden encaminarse las investigaciones. De manera general, se puede estar hablando de tres finalidades complementarias que tienen en cuenta el pasado, presente y futuro en una investigación, como se evidencia en la Figura 1.

Por otro lado, Randolph, (2009) y Snyder (2019) destacan la finalidad de la RL como posibilidad para comprender el estado actual del conocimiento sobre el objeto de estudio en relación con autores, metodologías, paradigmas y perspectivas, con lo que se evidencian vacíos y perspectivas de investigación en ese campo, para proponer agendas de investigación. 


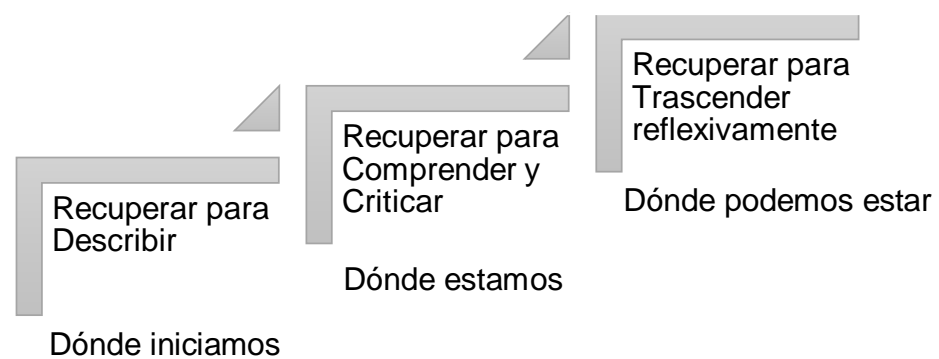

Fig. 1. Propósitos de la RL. Adaptada de Gómez y Sepúlveda (2008)

Ampliando un poco más estas finalidades, se pueden señalar algunos de los objetivos que proponen Guirao (2015), Talinbe y Owusu-Ansah (2014) y Hart (1998): 1) Identificar los aspectos relevantes conocidos, los desconocidos y los controvertidos sobre el tema de investigación. 2) Centrar el tema de interés y definir el alcance de una investigación. 3) Conocer las tendencias y perspectivas epistemológicas, metodológicas y disciplinares acerca del objeto de estudio. 4) Identificar aproximaciones teóricas y críticas. 5) Identificar las variables asociadas al estudio del tema y los instrumentos para su medición. 6) Identificar vacíos y necesidades, así como aciertos, desafíos y oportunidades en torno al campo del saber en cuestión. 7) Contextualizar los hallazgos de la investigación.

La RL desde el enfoque "semi-sistemático" de Snyder (2019) se puede considerar como una herramienta de investigación formativa que favorece el proceso investigativo de estudiantes para la formulación y desarrollo de proyectos de investigación, pues con ella se mapean y evalúan enfoques y temas teóricos y se identifican brechas de conocimiento en un campo disciplinar. También en Berns et al. (2018), Orozco y Díaz (2018), Posada (2017), Gómez et al. (2015) y Huergo (2015), se infiere este carácter formativo que tiene la RL para el estudiante, pues en el proceso investigativo de ésta, se desarrollan destrezas científicas como la documentación, la identificación, la clasificación, el análisis, la revisión crítica, el diseño de estrategias de recolección de datos, la comparación de métodos y resultados, además se descubren vacíos y se vislumbran posibilidades de estudio y solución de problemas. Además, con la RL el estudiante puede configurar una formación crítica, en el orden del conocimiento disciplinar, temático, metodológico y técnico para su ejercicio profesional (Talinbe y Owusu-Ansah, 2014 y Díaz et al., 2017).

Como complemento a lo precedente, y siguiendo el modelo metodológico que articula el campo de investigación en niveles y fases, Huergo (2015) asume la RL como una metodología de investigación, lo mismo que Snyder (2019), que puede calificarse como "formativa", en cuanto la investigación se desarrolla en la misma práctica investigativa de la búsqueda y revisión documental. Por ello, sumado a lo anterior, argumenta que en una investigación hay cuatro niveles fundamentales que configuran las partes esenciales de un proyecto de investigación donde la realización de cada uno de ellos no se da de manera aislado e independiente, pues estos niveles conforman un todo holístico que, como tal, requiere de un desarrollo progresivo y de retroalimentación. Por esta razón, investigar más que un acto aislado es un proceso secuencial, un camino que se construye paso a paso, donde cada nivel es fundamental para el desarrollo del siguiente. Sin embargo, el proceso solo nada indica, "si no está en íntima conexión con el colectivo de investigadores; este remite indudablemente a un equipo comprometido, donde lo teórico, lo práctico y lo contextual se conjugan con el ejercicio de profundos y reconocidos valores éticos" (Hoyos, 2000, p. 6).

Se dice que a investigar se aprende investigando, sin embargo, es complejo aprender solo, se requiere de un contexto donde el aprendizaje pueda darse de mejor calidad, el cual esté mediado por una comunidad académica en la que, con el ejemplo y la práctica, se va formando el investigador novel (Rojas, 2008), pues se aprende con otros investigadores y no por simple reflejo o mimetismo, sino, por la realización conjunta de trabajos concretos de investigación, en el marco organizativo de la internacionalización y con los recursos de instituciones, grupos concretos de investigación y redes de producción académica y científica (Kessing et al., 2017; Ortiz et al., 2012); no es un ejercicio anónimo y privado, sino público, es un quehacer social en el que está involucrada una comunidad científica (Sánchez, 2014). Por eso, como bien expresa Deslauriers (2004): "la ciencia es profundamente personal y también profundamente social; navegando entre la autobiografía y el distanciamiento, cada uno encuentra su equilibrio" (p. 25), o como el mismo Sánchez (2014) argumenta: "acompañar y "hacer el camino" aliado del investigador novel parece más efectivo y fecundo, que establecer ante él el camino idealizado que debería seguir en su fatigoso y difícil propósito de construir conocimiento científico" (p. 58). Así pues, la investigación se aprende en la práctica, no solo para el desarrollo de un proyecto de grado, sino, asumiéndola como una actividad para desarrollar en la cotidianidad de la vida. Ya decían también Booth et al. (2001) en relación con este sentido de caminar con otros en el quehacer investigativo: "la investigación nunca es una tarea solitaria. Aun cuando parezca que trabaja solo, en realidad camina sobre las huellas de otros, beneficiándose de su obra, sus principios, sus prácticas" (p.91). 


\section{La revisión de literatura en las fases del proceso de investigación}

Se había mencionado ya que la RL es una artesanía intelectual, un "ir tras las huellas de...", en un entramado de caminos que se puede plantear como un laberinto, difícil pero siempre con posibilidad de encontrar una salida. Desde esta perspectiva, Bem (1995) señalan que una RL implica aproximarse a un laberinto de perspectivas epistemológicas y metodológicas, así como a posturas ideológicas y supuestos implícitos y explícitos donde el campo de investigación no es inteligible a simple vista.

Es común encontrar en los autores que fundamentan este escrito, una metodología heurística y hermenéutica para RL (Gómez et al., 2015; Barbosa et al., 2013, Jiménez, 2006; Hoyos, 2000). Heurística, del griego heuriskein significa descubrir, encontrar e indagar, en nuestro caso, en documentos y fuentes históricas. La heurística se asume como arte de la búsqueda, como técnica de la indagación, de la invención y del descubrimiento que reconoce todos los argumentos que puedan conducir a realizar los proyectos propuestos. "Es reenviando a las fuentes mismas de la reflexión metodológica sobre el conocimiento que podemos discernir la aparición de las primeras consideraciones heurísticas" (Mandolini, 2013, p. 67). Invención y descubrimiento que no se da por arte de magia o fruto del azar, sino del esfuerzo y la reflexión del investigador, donde ahora si puede exclamar el conocido: Eureka -lo encontré-.

Por otro lado, lo hermenéutico, hace explícita y directa la interpretación como proceso que acompaña de principio a fin el trabajo investigativo como interpretación de los textos y/o materiales, interpretación de significados, de ideas, de contextos y pretextos, que los mismos autores han expresado. De manera puntual Jiménez (2006) señala que se habla de la "necesidad hermenéutica de remitirnos a textos que a su vez son expresiones de desarrollos investigativos, dados desde diversas percepciones de las ciencias y escuelas de pensamiento" (p. 32). Este ejercicio hermenéutico está relacionado fundamentalmente con análisis de los datos y la fase tres del proceso de investigación.

En el discurso del método, Descartes señala que la razón es por naturaleza igual en todos los hombres, pero se interroga sobre el por qué o cómo la misma razón produce una diversidad de opiniones. Y precisamente argumentando sobre el método, dice que la diferencia de tal variedad "no viene de que unos seamos más razonables que otros, sino, del hecho que conducimos nuestros pensamientos por diversas vías, y no consideramos las mismas cosas" (Martínez-Miguélez, 2004, p. 17). Ahora bien, centremos la mirada en el proceso y las fases de la investigación en relación con la $R L$, reconociendo que de manera general la investigación cuantitativa y cualitativa poseen en común las mismas fases: a) de planeación o preparatoria, b) descriptiva, c) de interpretación y análisis, d) de construcción teórica, e) de socialización.

Si bien la RL es un momento puntual dentro del proceso metodológico, también se puede asumir como una modalidad investigativa que atraviesa todo el proceso y se realiza de manera paralela con todas las fases de la investigación (Díaz et al., 2017; Restrepo et al., 2014; Randolph, 2009), o ser ella misma una investigación con cuatro momentos: diseño, realización, análisis y redacción de la revisión (Snyder, 2019). Así pues, en la Figura 2 se observa la idea de la $R L$ como esa espiral de investigación, en la que se mezclan las dos comprensiones: como "momento del proceso metodológico" -indagar, identificar, sistematizar, analizar y construir- y como "modalidad de investigación" en relación con el proceso en sus cinco fases.

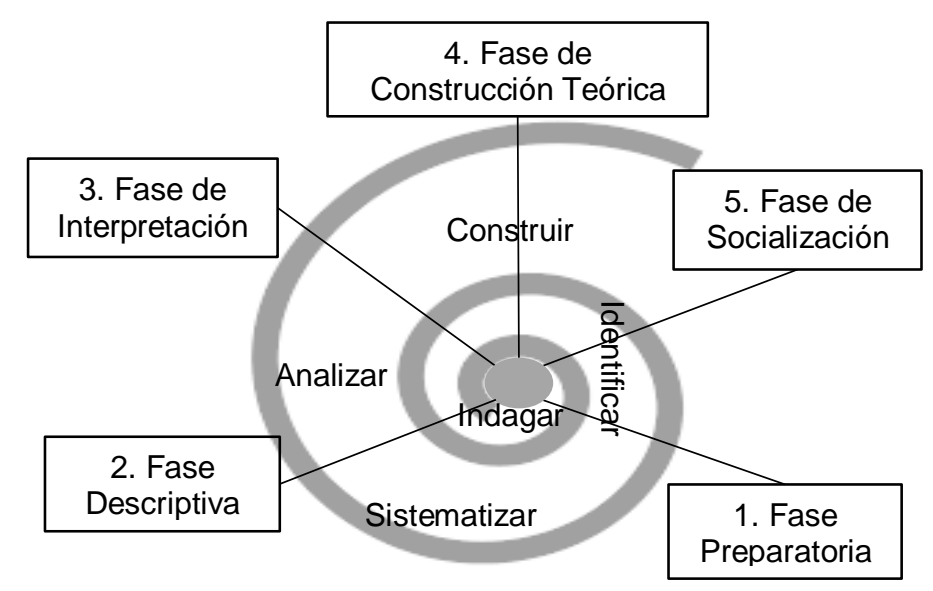

Fig. 2. La espiral de la $R L$ en el proceso de la investigación 


\section{Fase de planeación o preparatoria}

Esta fase busca orientar al investigador sobre la temática central y los subtemas que investigará, así como la cuestión metodológica, técnica, instrumental y contextual en que habrá de realizarse el estudio. En este sentido, es importante tener presente esta fase para comenzar la $R L$ habiendo determinado el problema de estudio. Sin embargo, siguiendo los argumentos de Esquivel (2013) sobre el 'estado de la cuestión', se puede inferir que la $R \mathrm{~L}$ contribuye también a delimitar dicho problema. Por tanto, es un requerimiento indispensable en la delimitación del tema, objeto y problema de estudio.

También implica el reconocimiento de los límites espacio temporales de lo que ya ha sido dado a conocer, para encontrar las preguntas inéditas, susceptibles de ser pensadas e investigadas desde lo atesorado en ese campo del conocimiento. Para iniciar una RL se requiere "la definición de un problema investigativo, y ante todo de "una pregunta" que, ubicada en una temática definida por el investigador, se convierte en el primer requisito para iniciar el rastreo y la pesquisa de información" (Jiménez, 2006, p. 36). O bien como señala Guirao (2015): "Dentro del proceso de investigación la revisión de la bibliografía se encuentra inicialmente en el proceso de justificación de la investigación para mostrar cuales son las metas, los objetivos, el alcance, y la exposición de motivos que fundamentan la investigación".

\section{Fase descriptiva}

Esta fase es llamada así por el resultado que consiste en el conjunto de fichas bibliográficas descriptivas de las unidades de análisis. Es decir, después de la indagación documental se describen los referentes disciplinares y teóricos de los ejes temáticos, así como los autores, sus contextos, sus perspectivas metodológicas y sus instrumentos o técnicas utilizadas (Calvo y Vélez, 1992). En esta fase son importantes dos herramientas: una "matriz bibliográfica" (Gómez et al., 2015) y un "diario del estado de la cuestión" (Esquivel, 2013), en el que se registren de manera organizada las consultas hechas, incluso, puede ser útil el uso de organizadores gráficos que sean de la experticia del investigador, reflexiones relevantes, inquietudes, registrar páginas electrónicas que despierten el interés, entro otros elementos.

\section{Fase de interpretación y análisis}

Se comprende como una labor hermenéutica en la que los elementos contextuales, epistemológicos y metodológicos, encontrados en la revisión documental, se ponen a dialogar con los resultados de la investigación, trascendiendo la fase puramente descriptiva para identificar y discutir sobre los núcleos temáticos o categorías de análisis. "La interpretación es fuente de nuevas constataciones y se nutre de las ya alcanzadas; para ser nuevamente constatada actúa como teoría, para nutrirse de las constataciones es pura hermenéutica" (Calvo y Vélez, 1992). Además, la RL contribuye para comparar resultados y conclusiones en estudios similares desde una mirada crítica y analítica (Guirao, 2015; Randolph, 2009).

\section{Fase de construcción teórica}

Como fruto del ejercicio hermenéutico acerca de las categorías de análisis, en esta fase se genera propiamente un nuevo estado del conocimiento sobre el objeto de estudio que permite orientar nuevas líneas o procesos de investigación. Para esta fase se pueden enunciar tres puntos abordados por Restrepo et al. (2014), permite orientar la construcción de nuevas líneas de investigación, generar comprensiones sobre las formas de abordaje analítico de fenómeno estudiado y crear hipótesis teóricas que explican la manera como han sido abordados los temas/problemas de estudio. El estudio realizado por Tan (2007) expone que la experiencia de los estudiantes en esta etapa es emocionante; ellos manifestaban ansias por encontrar la solución al problema planteado, después de sintetizar los resultados, escribir las conclusiones que fueron aprobadas por sus mentores.

\section{Fase de socialización y publicación}

Consiste en la divulgación de los resultados en forma oral o escrita, ya sea a través de conferencias, seminarios, simposios, talleres, publicación de artículo, capítulo de libro o libro. "La importancia de esta fase estriba en poner en circulación un nuevo conocimiento que permite la interlocución con otros grupos y comunidades científicas" (Guevara, 2016). En esta fase el investigador marca su huella que otros podrán mirar para guiar sus trabajos, para seguir trasegando el sendero de la investigación. Si bien esta es la meta de la investigación científica, se requiere de un proceso de acompañamiento, de mentoría desde la investigación formativa que realicen investigadores ya consolidados, para alcanzar dicho fin, como señalan Rojas y Méndez (2017) y Díaz et al (2017). 
Las experiencias narradas en el estudio de Tan (2007) muestra la relevancia de esta última fase del proceso de investigación pues los estudiantes señalan que se gana reconocimiento con su trabajo. Algunos tuvieron la oportunidad de presentarlos en espacios académico y otros lograron publicar en revistas arbitradas. Por eso las RL deben tener profundidad y rigor científico como señala Palmatier et al. (2018).

Así pues, se puede colegir que la $R L$ en las fases de contribuye a partir de estas fases de un proyecto de investigación a la formación para la investigación, y de forma más importante para la formación de investigadores. A través de esta práctica se despliegan capacidades para el buen desarrollo del ejercicio investigativo: para delimitar el problema y el alcance de la investigación, para la búsqueda y desarrollo de herramientas teóricas y metodológicas, para la organización y procesamiento de la información, para la inferencia de los posibles resultados, para el análisis y la confrontación de lo investigado, así como para proyectar nuevos trabajos complementarios (Guevara, 2016).

\section{CONCLUSIONES}

De acuerdo con el trabajo presentado y a los resultados obtenidos, se pueden plantear las siguientes conclusiones principales:

El primer camino que realiza el investigador para iniciar un proyecto es la RL; a través de este recorrido, se forma en un intercambio de información estableciendo una relación académica con diferentes autores y con un objeto de estudio, lo cual contribuye a definir el alcance de su investigación.

Con la $R L$ se obtiene el material necesario para problematizar el fenómeno de estudio, para formular hipótesis y propuestas de acción que más adelante servirán también en el campo conceptual. Pero también de un marco teórico se pueden deducir los objetivos de una investigación.

La RL es el sendero que permite el estudio del conocimiento acumulado y sus orígenes. Es el punto de partida de una metodología de contextualización, clasificación y categorización. En este sentido, investigar a partir de una RL no puede ser un acto aislado, sino un proceso que implica secuencialidad y ciclicidad en las fases, pues cada paso es útil para la construcción del siguiente y para retroalimentar los anteriores.

La RL no puede ser única y cerrada, pues la complejidad e incertidumbre de lo que se vaya a encontrar en los datos, requiere de una revisión permanente, sostenida y abierta de literatura relacionada con el tema o problema de estudio, la cual se constituye en un referente que sirve de guía indicativa y provisional para apoyar la construcción conceptual.

Finalmente, después de este recorrido hermenéutico pedagógico sobre la $R L$, consideramos su importancia como una posibilidad para incursionar en lo desconocido, incluso se pudiera decir que para innovar en el desarrollo del conocimiento, pues si bien ya existe un conocimiento determinado sobre una temática o realidad social, sin embargo, no está dicha la última palabra, siempre hay que indagar, hay que transitar hacia lo inédito, buscando en lo no transitado las respuestas que esperan ser encontradas o simplemente resignificadas, por supuesto, a partir de las huellas que otros han dejado.

\section{REFERENCIAS}

Akerlind, G.S., Variation and commonality in phenomenographic research methods, https://doi.org/10.1080/07294360500284672, Higher education research \& development, 24(4), 321-334 (2005)

Barattucci, M. y Bocciolesi, E., Phenomenography in the Student Learning Perspective: A Review of Studies in Academic Contexts, https://doi.org/10.6092/issn.1825-8670/7900, Encyclopaideia - Journal of Phenomenology and Education, 22(50), 21-34 (2018)

Barbosa, J.W., Barbosa, J.C. y Rodríguez, M., Revisión y análisis documental para estado del arte: una propuesta metodológica desde el contexto de la sistematización de experiencias educativas, http://dx.doi.org/10.1016/S0187358X(13)72555-3, Investigación bibliotecológica, 27(61), 83-105 (2013)

Barczak, G., Writing a review article, https://doi.org/10.1111/jpim.12365, The Journal of Product Innovation Management, 34(2), 120-121 (2017)

Bem, D.J., Writing a review article for psychological bulletin, https://doi.org/10.1037/0033-2909.118.2.172, Psychological Bulletin, 118(2), 172-177 (1995)

Bettencourt, L.A. y Houston, M:B., The impact of article method type and subject area on article citations and reference diversity in JM, JMR, and JCR, https://doi.org/10.1023/A:1012272305777, Marketing Letters, 12(4), 327-340 (2001)

Booth, W., Colomb, G. y Williams, J., Cómo convertirse en un hábil investigador, $1^{1}$ edición, Editorial Gedisa, Barcelona, España (2001) 
Berns, A., Palomo, M., Calderón, A. y Dodero, J., Iniciar al alumnado de Estudios Ingleses en la investigación científica a partir de TFGs centrados en revisiones sistemáticas de la literatura, https://doi.org/10.4995/redu.2018.10188, REDU. Revista de Docencia Universitaria. 16(2),143-158 (2018)

Calvo, G. y Vélez, A., Análisis de la investigación en la formación de investigadores: nueve años de la maestría en educación en la Universidad de la Sabana -el estado del arte o del conocimiento-, Universidad de La Sabana, Bogotá, Colombia (1992)

Deslauriers, J.P., Investigación cualitativa. Guía práctica, Editorial Papiro, Pereira, Colombia (2004)

Díaz, O., Montes, M. y Cangahuala, O., La investigación formativa en el pregrado: una propuesta desde el plan de estudios de la Facultad de Ciencias Contables de la PUCP, http://dx.doi.org/10.21710/rch.v19i0.358, Hermes, 19, 409-431 (2017)

Esquivel, F., Lineamientos para diseñar un estado de la cuestión en investigación educativa, https://doi.org/10.15517/revedu.v37i1.10631, Revista Educación, 37(1), 65-87 (2013)

Flórez, R., Hacía una pedagogía del conocimiento. McGraw Hill, Bogotá, Colombia (1997)

Gallego, L. y Araque, O., Variables de influencia en la capacidad de aprendizaje. un análisis por conglomerados y componentes principales, http://dx.doi.org/10.4067/S0718-07642019000200257, Información Tecnológica, 30(2), 257-264 (2019)

Guirao, S.J.A., Utilidad y tipos de revisión de literatura, https://dx.doi.org/10.4321/S1988-348X2015000200002, ENE. Revista de Enfermería, 9(2), (2015)

Gómez, M.E. y Sepúlveda, M.P., Evaluar el estado del arte de la aplicación de la producción más limpia como herramienta estratégica en la solución de los problemas o conflictos ambientales en el sector industrial a nivel global, nacional, regional y territorial en los últimos cuatro años. (Tesis de especialización inédita), Universidad de Antioquia, Medellín, Colombia (2008)

Gómez, M., Galeano, C. y Jaramillo, D.A., El estado del arte: una metodología de la investigación, https://doi.org/10.21501/22161201.1469, Revista Colombiana de Ciencias Sociales, 6(2), $423-442$ (2015)

Guevara, R., El estado del arte en la investigación: ¿análisis de los conocimientos acumulados o indagación por nuevos sentidos?, https://doi.org/10.17227/01234870.44folios165.179, Folios, 44, 165-179 (2016)

Hart, C., Doing a literature review: Releasing the social science research imagination, Sage, London, England (1998)

Hoyos, C., Un modelo para investigación documental: guía teórico-práctica sobre construcción de Estados del Arte con importantes reflexiones sobre la investigación, Señal Editora, Medellín, Colombia (2000)

Huber, J., Kamakura, W. y Mela, C.F., A topical history of JMR, https://doi.org/10.1509/jmr.51.1.02, Journal of Marketing Research, 51(1), 84-91 (2014)

Huergo, P.L., Importancia y pasos para la elaboración del estado del arte en un anteproyecto o proyecto de investigación, http://dx.doi.org/10.16925/greylit.1073, Documento de docencia, 1(2) 1-23 (2015)

Hult, G.T.M., JAMS 2010-2015: literature themes and intellectual structure, https://doi.org/10.1007/s11747-015-0464-3, Journal of the Academy of Marketing Science, 43(6), 663-669 (2015)

Mendoza, V. y Jaramillo, S.S., Guía para la elaboración de ensayos de investigación, https://www.redalyc.org/articulo.oa?id=342/34202605, 1405-6690, Revista del Centro de Investigación. Universidad La Salle, 7(26), 63-79 (2006)

Jiménez, A., El estado del arte en la investigación en ciencias sociales. En A. Torres, A. y Jiménez, A., La práctica investigativa en ciencias sociales (pp. 27-42), Universidad Pedagógica Nacional, Bogotá, Colombia (2006)

Jones, T.M., Wicks, A.C. y Freeman, R.E., Stakeholder theory: the state of the art, https://doi.org/10.1002/9781405164771.ch1, The Blackwell Guide to Business Ethics, 17-37 (2017)

Kessing, D., Denollet, J., Widdershoven, J. y Kupper, N., Self-care and health-related quality of life in chronic heart failure: A longitudinal analysis, https://doi.org/10.1177/1474515117702021, European Journal of Cardiovascular Nursing, 16(7), 605-613 (2017)

Kitchenham, B., Procedures for Performing Systematic Reviews, Keele University Technical Report TR/SE-0401, http://www.inf.ufsc.br/ aldo.vw/kitchenham.pdf, 1353-7776, 1-33 (2004)

Mandolini, R., Heurística y arte: una contribución para la comprensión de los procesos artísticos creativos, https://doi.org/10.22370/rhv.2013.1.85, Revista de Humanidades de Valparaíso, 1(1), 63-92 (2013)

Martínez-Miguélez, M., Ciencia y arte en la metodología cualitativa, Editorial Trillas, México (2004)

Olson, J.S., Wang, D., Olson, G.M. y J. Zhang, J., How people write together now: beginning the Investigation with advanced undergraduates in a project course, https://doi.org/10.1145/3038919, ACM transactions in computer-human interaction, 24(1), 1-40 (2017)

Orozco, J. y Díaz, A., ¿Cómo redactar los antecedentes en una investigación cualitativa? https://doi.org/10.30698/recsp.v1i2.13, Revista Electrónica de Conocimientos, Saberes y Prácticas, 1(2), 66-82 (2018) 
Ortiz, V., Moreno, M.G., Jiménez, J.M. y Barragán, B. M., Propuestas para la formación y desarrollo de Investigadores universitarios mexicanos, https://doi.org/10.35362/rie6011333, Revista Iberoamericana de Educación, 60(1), (2012)

Palmatier, R.W., Houston, M.B. y Hulland, J., Review articles: Purpose, process, and structure, https://doi.org/10.1007/s11747-017-0563-4, Journal of the Academy of Marketing Science, 46, 1-5 (2018)

Posada, N., Algunas nociones y aplicaciones de la investigación documental llamada estado del arte, http://dx.doi.org/10.22201/iibi.24488321xe.2017.73.57855, Investigación Bibliotecológica, 31(73), 237-263 (2017)

Pradhan, S. y Pradhan, R.K., An empirical investigation of relationship among transformational leadership, affective organizational commitment and contextual performance, https://doi.org/10.1177/0972262915597089, Vision: The Journal of Business Perspective, 19(3), 227-235 (2015)

Randolph, J.J., A guide to writing the dissertation literature review, Practical Assessment, https://doi.org/10.7275/b0az8t74, Research \& Evaluation, 14(13), 1-13 (2009)

Randolph, J.J., Multidisciplinary methods in educational technology research and development, HAMK Press, Hämeenlinna, Finland (2008)

Restrepo, A., Tabares, C., Tangarife, A.M. y Londoño, J., Los estados del arte y la producción de conocimiento en las Ciencias Sociales. Una mirada a la Ciencia Política, https://revistas.udea.edu.co/index.php/RIB/article/view/20522, 01200976, Revista Interamericana de Bibliotecología, 37(3), 227-236 (2014)

Rodríguez, S.P. y Acosta, W.A., La emergencia de la didáctica de las ciencias sociales: ¿campo en consolidación o en disgregación?, https://doi.org/10.17227/01234870.25folios37.52, Folios. 25, 37-52 (2007)

Rojas, M., El problema regional de la Formación de Jóvenes investigadores en el nivel de pregrado en las instituciones de educación superior del departamento de Tolima, (Tesis de Doctorado), http://bibliotecavirtual.clacso.org.ar/Colombia/alianza-cinde-umz/20091210034819/tesis-rojas.pdf, Centro de Estudios Avanzados en Niñez y Juventud alianza de la Universidad de Manizales y el CINDE, Colombia (2008)

Rojas, M. y Méndez, R., Procesos de formación en investigación en la universidad: ¿qué les queda a los estudiantes?, https://doi.org/10.18634/sophiaj.13v.2i.261, Sofhia, 13(2), 53-69 (2017)

Rubio, M.J., Torrado, M., Quirós, C. y Valls, R., Autopercepción de las competencias investigativas en estudiantes de último curso de Pedagogía de la Universidad de Barcelona para desarrollar su Trabajo de Fin de Grado, https://doi.org/10.5209/RCED.52443, Revista Complutense de Educación, 29 (2), 335-354 (2018)

Sánchez, R., Enseñar a investigar. Una didáctica nueva de la investigación en ciencias sociales y humanas, Universidad Nacional Autónoma de México-Instituto de Investigaciones sobre la Universidad y la Educación, México (2014)

Sánchez, H.H., La investigación formativa en la actividad curricular, https://doi.org/10.25176/RFMH.v17.n2.836, Rev. Fac. Med. Hum. 17(2), 71-74 (2017)

Snyder, H., Literature review as a research methodology: an overview and guidelines, https://doi.org/10.1016/j.jbusres.2019.07.039, Journal of Business Research, 104, 333-339 (2019)

Sorescu, A., Warren, N.L. y Ertekin, L., Event study methodology in the marketing literature: an overview, https://doi.org/10.1007/s11747-017-0516-y, Journal of the Academy of Marketing Science, 45(2), 186-207 (2017)

Talinbe, R., Owusu-Ansah, A., Essential ingredients of a Good research proposal for undergraduate and postgraduate students in social sciences, https://doi.org/10.1177/2158244014548178, SAGE Open, 4(3), 1-14 (2014)

Tan, E.R., Research Experiences of Undergraduate Students at a Comprehensive University, http://www.isetl.org/ijtlhe/pdf/lJTLHE158.pdf, 1812-9129, International Journal of Teaching and Learning in Higher Education, 19(3), 205-215 (2007)

Viteri, T.A., y Vázquez, S., Formación de habilidades de investigación formativa en los estudiantes de la carrera de ingeniería comercial de la Facultad de Ciencias Administrativas de la Universidad de Guayaquil, https://rus.ucf.edu.cu/index.php/rus/article/view/302, 2218-3620, Revista Universidad y Sociedad, 8(1), 36-44 (2016) 